\title{
Relationship between Estradiol Level, Transvaginal Ultrasound Endometrial Thickness, Body Mass Index and Endometrial Pathology in Women with Postmenopausal Bleeding
}

\author{
${ }^{1}$ Mohammed Samir Fouad Khalaf, ${ }^{1}$ Mahmoud Samy Zaki, ${ }^{2}$ Mohamed Saied Elshourbgy, \\ ${ }^{1}$ Ahmed Magdy Lotfy Abdelmageed \\ ${ }^{1}$ Department of Obstetrics and Gynecology, ${ }^{2}$ Department of Clinical Pathology, Faculty of Medicine, \\ Al-Azhar University
}

Corresponding author: Ahmed Magdy Lotfy Abdelmageed

\begin{abstract}
:
Background: menopause is derived from the Greek words; men (month) and pauses (Cessation). A woman is considered menopausal after cessation of menstruation for 1 year after an average age of 46 . Postmenopausal bleeding (PMB) is defined as bleeding recurring in a menopausal woman at least 1 year after cessation of cycles. It must always be investigated because many causes are premalignant or malignant. The most common premalignant and malignant causes are complex hyperplasia with atypia and carcinoma of the endometrium. These disorders are present in as many as $1 / 3$ of the patients evaluated for PMB in many series. Objective: this study aimed to explore the relationship between estradiol level, transvaginal ultrasound, endometrial thickness and body mass index and their correlation to endometrial pathology in women with postmenopausal bleeding. Patients and Methods: in the present study, 80 patients with postmenopausal bleeding were examined regarding BMI, ultrasonographic assessment of endometrial thickness and pathological findings of the endometrium. D\&C was performed for all patients under general anesthesia using a sharp ended curette starting first with the fundus, posterior wall, anterior wall, right and left lateral wall. The patients' age ranged from 46-69 years, with a mean of $53.53 \pm 4.1$ years. Results: in the present study, there was a highly statistically significant difference between benign and malignant groups as the endometrium was markedly thicker in the malignant group. Our study displayed that there was a statistically high significant difference between benign and malignant groups as regard the presence of fibroid uterus (due to presence of excess unopposed estrogen in those obese women) and the presence of adenomyotic or normal uterus inbetween both groups. Conclusion: transvaginal ultrasonography is an excellent firststep diagnostic method of excluding the endometrial abnormalities in women with postmenopausal bleeding. Ideally, a non-invasive investigation is preferred over an invasive one and also an economical investigation preferred over an expensive one; this applies equally to affluent countries and third world. Endometrial biopsy is the "gold standard" for diagnosing abnormalities in the endometrial tissue of patients with PMB.
\end{abstract}

Keywords: postmenopausal bleeding, transvaginal ultrasound, relative risk.

\section{INTRODUCTION}

Postmenopausal bleeding (PMB) is the occurrence of vaginal bleeding following a woman's last menstrual cycle. Accounts for five per cent of office gynaecology presentations were detected. Generally, four to 11 per cent of postmenopausal women will experience bleeding. The chance of this occurring reduces as time since menopause increases ${ }^{(\mathbf{1})}$.The majority of women referred to outpatient gynaecological services had pelvic ultrasound in order to evaluate the endometrial thickness and assess for pelvic pathology. Transvaginal ultrasound (TVUS) is considered an acceptable initial investigation in women with PMB ${ }^{(1)}$. The association between obesity and endometrial cancer was greater in postmenopausal women compared to women of reproductive age, and also in non users of COCs had a higher risk, obesity increases the relative risk (RR) of developing endometrial cancer two- to threefold ${ }^{(2)}$.

An endometrial biopsy is considered the gold standard for evaluation of PMB. Endometrial biopsy can be obtained with an endometrial pipelle in the outpatient setting, or by hysteroscopy and curettage (with or without dilatation) in either the outpatient or inpatient setting ${ }^{(3)}$. 


\section{AIM of the WORK}

The current study was designed to explore the relationship between estradiol level, transvaginal ultrasound, endometrial thickness and body mass index and their correlation to endometrial pathology in women with postmenopausal bleeding.

\section{PATIENTS and METHODS}

The present study was a prospective cross sectional study which was conducted on 80 patients with postmenopausal bleeding.

Inclusion criteria: menopausal women (defined as absence of menstruation for at least 1 year after the age of 45 years provided that the amenorrhea was not explained by pregnancy, medication or disease), bleeding after menopause $\geq 12$ months and endometrial thickness by TVUS $\geq 5 \mathrm{~mm}$.

Exclusion criteria: bleeding after menopause less than 12 months, hormone replacement therapy and tamoxifen users, chronic diseases as diabetes mellitus or hypertension, history of hematological disorders, history of anticoagulant therapy , obvious causes of bleeding from cervix or vagina e.g. ulcer and Adnexal masses (with suspicious of malignancy) detected by TVUS.

\section{Methodology in details:}

All patients were subjected to the following: Informed verbal consent: it was obtained from all women included in the study. The study was approved by the Ethics Board of Al-Azhar University.

Full medical history: personal history: current age, age at time of menopause, and parity, present history of bleeding including onset, course, duration and criteria of bleeding pattern, history of use of particular drug intake, history of diabetes mellitus or hypertension, past history of bleeding tendency, blood diseases, history of any operative interference and similar conditions and family history of similar condition.

General examination: including: weight, height and body mass index (BMI) (dividing the patient's weight in $\mathrm{kg}$ by the square of her height in meters), vital signs (blood pressure and pulse), pallor and signs of anemia, cardiac examination, chest examination and abdominal examination.

Local examination: including: Inspection of external genitalia, bimanual examination to detect uterine size, mobility, tenderness and adnexal masses, speculum examination for cervical lesions, erosions, hypertrophy and ulcers or vaginal lesions.

Laboratory investigations: included: complete blood count, coagulation profile, fasting and postprandial blood sugar, liver and kidney functions tests and measurement of estradiol level.

Transvaginal ultrasound: it was carried out by using MEDISON SONOACE X4 EXP. equipped with 5-7 $\mathrm{MHz}$ transvaginal transducer. The bladder was completely emptied and the procedure was explained to the patient. The patient then placed in a dorsal recumbent position and draped, then the transducer was put in a rubber glove, covered with gel and inserted into the vagina.

This study assessed the following: measurement of the longitudinal, transverse and anteroposterior diameters of the uterus, endometrial thickness, endometrium was scanned from cornu to cornu then endometrial thickness was measured at its thickest point between the two basal layers on the anterior and posterior uterine walls in the sagittal plane of the uterus, callipers were placed perpendicular to the outer edge of the endometrium. If there was fluid in the endometrial cavity, the endometrial thickness was measured as above but with the inclusion of the cavity fluid and the double endometrial stripe and then the fluid diameter was subtracted at the same point. Any focal lesion such as: myoma, adenomyosis, endometrial polyp were reported. The adnexae were examined for the presence of any pathology.

Conventional dilatation and curettage: the first sample was taken from endocervical canal before dilatation, then cervical dilatation up to 7-8 Hegar. A sharpended curette was introduced and curettage starting first with fundus then posterior wall then anterior wall then right then left lateral walls, if polyp was present we used ovum forceps to grasp it. The specimen was placed in formalin $10 \%$ until histopathological examination. According to the histopathology of the studied patients, they were classified into 2 groups: the benign group which included hyperplasia without atypia, hyperplastic endometrial polyp, disordered proliferative endometrium, atrophic endometrium. The malignant group which included hyperplasia with atypia and endometrial carcinoma.

\section{Statistical analysis}


Relationship between Estradiol Level, Transvaginal Ultrasound Endometrial Thickness...

The Data were collected and entered into the personal computer. Statistical analysis was done using Statistical Package for Social Sciences (SPSS/version 22) software.

\section{RESULTS}

Table (1): Comparison between the two studied groups regarding age

\begin{tabular}{|l|c|c|c|}
\hline & Benign & Malignant & Total \\
\hline Age & & & \\
Range & $46.0-58.0$ & $52.0-69.0$ & $46.0-69.0$ \\
Mean & 52.58 & 59.45 & 53.53 \\
S.D. & 2.95 & 5.37 & 4.10 \\
\hline $\mathbf{t}(\mathbf{p})$ & \multicolumn{3}{|c|}{$19.25\left(0.0001^{*}\right)$} \\
\hline
\end{tabular}

t: Student t-test

$\mathrm{p}$ : $\mathrm{p}$ value for comparing between the studied groups

* Significant at level 0.05

Table (2): Comparison between the two studied groups regarding gravidity and parity

\begin{tabular}{|l|c|c|c|}
\hline & Benign & Malignant & Total \\
\hline Gravidity & 3.0 & 2.0 & 2.0 \\
Range & 7.0 & 5.0 & 7.0 \\
Mean & 4.80 & 3.73 & 4.65 \\
S.D. & 1.32 & 1.10 & 1.34 \\
\hline t(p) & \multicolumn{3}{|c|}{} \\
\hline Parity & 3.0 & $2.449\left(0.013^{*}\right)$ & 2.0 \\
Range & 7.0 & 2.0 & 7.0 \\
Mean & 4.54 & 4.0 & 4.29 \\
S.D. & 1.09 & 2.73 & 1.22 \\
\hline t(p) & \multicolumn{3}{|l}{} \\
\hline
\end{tabular}

t: Student t-test

$\mathrm{p}$ : $\mathrm{p}$ value for comparing between the studied groups

* Significant at level 0.05

Table (3): Comparison between the two studied groups regarding age at menopause and duration of PMB

\begin{tabular}{|l|c|c|c|}
\hline & Benign & Malignant & Total \\
\hline Age at menopause & 45.0 & 47.0 & 45.0 \\
Range & 54.0 & 55.0 & 55.0 \\
Mean & 49.30 & 51.09 & 49.55 \\
S.D. & 2.23 & 2.55 & 2.34 \\
\hline $\mathbf{t}(\mathbf{p})$ & \multicolumn{3}{|c|}{} \\
\hline Duration of PMB & 1.0 & $5.01\left(0.003^{*}\right)$ & 1.0 \\
Range & 10.0 & 2.0 & 19.0 \\
Mean & 3.28 & 19.0 & 3.98 \\
S.D. & 2.13 & 8.36 & 3.27 \\
\hline $\mathbf{t}(\mathbf{p})$ & \multicolumn{3}{|c|}{} \\
\hline
\end{tabular}

t: Student t-test

$\mathrm{p}$ : $\mathrm{p}$ value for comparing between the studied groups

* Significant at level 0.05Table (4): Comparison between the two studied groups regarding BMI

\begin{tabular}{|c|c|c|c|}
\hline & Benign & Malignant & Total \\
\hline BMI & 28.0 & 29.7 & 28.0 \\
Range & 36.9 & 44.8 & 44.8 \\
Mean & 32.38 & 36.85 & 32.99 \\
S.D. & 2.57 & 4.67 & 3.30 \\
\hline \multicolumn{2}{|c|}{} \\
\hline
\end{tabular}

t: Student t-test

$\mathrm{p}$ : $\mathrm{p}$ value for comparing between the studied groups

* Significant at level 0.05

Table (5): Comparison between the two studied groups regarding estradiol level

\begin{tabular}{|l|l|l|l|}
\hline & Benign & Malignant & Total \\
\hline
\end{tabular}




\begin{tabular}{|l|c|c|c|}
\hline Estradiol & 10.0 & 3.0 & 3.0 \\
Mean & 22.22 & 9.18 & 20.43 \\
S.D. & 3.17 & 2.95 & 9.08 \\
\hline $\mathbf{t}(\mathbf{p})$ & \multicolumn{3}{|c|}{$15.25(0.001 *)$} \\
\hline
\end{tabular}

t: Student t-test

$\mathrm{p}$ : $\mathrm{p}$ value for comparing between the studied groups

* Significant at level 0.05

Table (6): Comparison between the two studied groups regarding endometrial thickness

\begin{tabular}{|l|c|c|c|}
\hline & Benign & Malignant & Total \\
\hline Endometrial thickness & 6.0 & 21.3 & 6.0 \\
Mean & 11.84 & 33.35 & 14.80 \\
S.D. & 3.83 & 8.06 & 4.74 \\
\hline $\mathbf{t}(\mathbf{p})$ & \multicolumn{3}{|c|}{$12.65\left(0.001^{*}\right)$} \\
\hline
\end{tabular}

t: Student t-test

$\mathrm{p}$ : $\mathrm{p}$ value for comparing between the studied groups

* Significant at level 0.05

Table (7): Comparison between the two studied groups regarding ultrasonographic findings

\begin{tabular}{|c|c|c|c|c|}
\hline \multirow{2}{*}{\multicolumn{2}{|c|}{ Ultrasonographic findings }} & \multicolumn{2}{|c|}{ Group } & \multirow{3}{*}{$\begin{array}{c}\text { Total } \\
50\end{array}$} \\
\hline & & Benign & Malignant & \\
\hline \multirow{2}{*}{ Normal } & No. & 50 & 0 & \\
\hline & $\%$ & $72.5 \%$ & $0.0 \%$ & $62.5 \%$ \\
\hline \multirow{2}{*}{ Adenomyosis } & No. & 14 & 5 & 19 \\
\hline & $\%$ & $20.3 \%$ & $45.5 \%$ & $23.8 \%$ \\
\hline \multirow{2}{*}{ Fibroid } & No. & 5 & 6 & 11 \\
\hline & $\%$ & $7.2 \%$ & $54.5 \%$ & $13.8 \%$ \\
\hline \multirow{2}{*}{ Total } & No. & 69 & 11 & 80 \\
\hline & $\%$ & $100.0 \%$ & $100.0 \%$ & $100.0 \%$ \\
\hline \multicolumn{2}{|c|}{$\mathbf{X}^{2}(\mathbf{p})$} & \multicolumn{2}{|c|}{$27.005(0.0001 *)$} & \\
\hline
\end{tabular}

$\chi^{2}:$ Chi square test

$\mathrm{p}$ : $\mathrm{p}$ value for comparing between the studied groups

* Significant at level 0.05

Table (8): Comparison between the two studied groups regarding ultrasound endometrial margin

\begin{tabular}{|c|c|c|c|c|}
\hline \multirow{2}{*}{\multicolumn{2}{|c|}{ Ultrasound endometrial margin }} & \multicolumn{2}{|c|}{ Group } & \multirow{2}{*}{ Total } \\
\hline & & Benign & Malignant & \\
\hline \multirow{2}{*}{ Regular } & No. & 62 & 3 & 65 \\
\hline & $\%$ & $89.9 \%$ & $27.3 \%$ & $81.2 \%$ \\
\hline \multirow{2}{*}{ Irregular } & No. & 7 & 8 & 15 \\
\hline & $\%$ & $9.1 \%$ & $72.7 \%$ & $18.7 \%$ \\
\hline \multirow{2}{*}{ Total } & No. & 69 & 11 & 80 \\
\hline & $\%$ & $100.0 \%$ & $100.0 \%$ & $100.0 \%$ \\
\hline \multicolumn{2}{|c|}{$\mathbf{X}^{2}(\mathbf{p})$} & \multicolumn{2}{|c|}{$22.427(0.0001 *)$} & \\
\hline
\end{tabular}

$\chi^{2}$ : Chi square test

$\mathrm{p}$ : $\mathrm{p}$ value for comparing between the studied groups

* Significant at level 0.05

Table (9): Comparison between the two studied groups regarding ultrasound endometrial echogenicity

\begin{tabular}{|c|c|c|c|c|}
\hline \multicolumn{2}{|c|}{ Ultrasound endometrial echogenicity } & \multicolumn{2}{|c|}{ group } & \multirow{2}{*}{ Total } \\
\cline { 2 - 5 } \multirow{2}{*}{ Homogenous } & No. & Benign & Malignant & 35 \\
\cline { 2 - 5 } & $\%$ & $50.7 \%$ & 0 & $43.8 \%$ \\
\hline \multirow{2}{*}{ Heterogenous } & No. & 34 & $0.0 \%$ & 45 \\
\cline { 2 - 5 } & $\%$ & $49.3 \%$ & $100.0 \%$ & $56.2 \%$ \\
\hline \multirow{2}{*}{ Total } & No. & 69 & 11 & 80 \\
\cline { 2 - 5 } & $\%$ & $100.0 \%$ & $100.0 \%$ & $100.0 \%$ \\
\hline \multicolumn{2}{|c|}{$14.010(0.001 *)$} & \\
\hline
\end{tabular}

$\chi^{2}$ : Chi square test

$\mathrm{p}$ : $\mathrm{p}$ value for comparing between the studied groups

* Significant at level 0.05

Table (10): Comparison between the two studied groups regarding endometrial pathology 
Relationship between Estradiol Level, Transvaginal Ultrasound Endometrial Thickness...

\begin{tabular}{|l|c|c|c|c|}
\hline \multicolumn{2}{|c|}{} & Benign & Malignant & \\
\hline \multirow{2}{*}{ Endometrial hyperplasia without atypia } & No. & 34 & 0 & 34 \\
\cline { 2 - 4 } & $\%$ & $49.3 \%$ & $0.0 \%$ & $42.5 \%$ \\
\hline \multirow{2}{*}{ isordered proliferative } & No. & 15 & 0 & 15 \\
\cline { 2 - 5 } & $\%$ & $21.7 \%$ & $0.0 \%$ & $18.8 \%$ \\
\hline \multirow{2}{*}{ Hyperplastic Endometrial polyp } & No. & 16 & 0 & 16 \\
\cline { 2 - 5 } & $\%$ & $23.2 \%$ & $0.0 \%$ & $20.0 \%$ \\
\hline \multirow{2}{*}{ Atrophic endometrium } & No. & 4 & 0 & 4 \\
\hline \multirow{2}{*}{ Endometrial carcinoma } & $\%$ & $5.8 \%$ & $0.0 \%$ & $5.0 \%$ \\
\hline \multirow{2}{*}{ Hyperplasia with atypia } & No. & 0 & 5 & 5 \\
\hline \multirow{2}{*}{ Total } & No. & $0.0 \%$ & $45.5 \%$ & $6.2 \%$ \\
\cline { 2 - 5 } & $\%$ & $0.0 \%$ & 6 & 6 \\
\cline { 2 - 5 } & No. & 69 & $54.5 \%$ & $7.5 \%$ \\
\hline
\end{tabular}

Table (11): Comparison between the two studied groups regarding endometrial polyp

\begin{tabular}{|c|c|c|c|c|}
\hline \multicolumn{2}{|c|}{ Endometrial polyp } & \multicolumn{2}{c|}{ group } & \multirow{2}{*}{ Total } \\
\cline { 3 - 5 } & Benign & Malignant & \\
\hline \multirow{2}{*}{ Endometrial polyp } & No. & 15 & 0 & 15 \\
\cline { 2 - 5 } & $\%$ & $21.7 \%$ & $0.0 \%$ & $18.8 \%$ \\
\hline \multirow{2}{*}{ No endometrial polyp } & No. & 54 & 11 & 65 \\
\cline { 2 - 5 } & $\%$ & $78.3 \%$ & $100.0 \%$ & $81.2 \%$ \\
\cline { 2 - 5 } Total & No. & 69 & 11 & $100.0 \%$ \\
\hline \multicolumn{2}{|c|}{} \\
\cline { 2 - 5 }
\end{tabular}

$\chi^{2}$ : Chi square test

$\mathrm{p}$ : $\mathrm{p}$ value for comparing between the studied groups

N.S.: not Significant

\section{DISCUSSION}

Malignancy is more common with postmenopausal bleeding than perimenopausal; it still the most common cause of postmenopausal bleeding is atrophic vaginitis or endometritis. Menopause is one of the critical periods of a women's life during which weight gain and onset or worsening of obesity are favoured. At this period the prevalence of obesity is the highest ${ }^{(4)}$.

As AUB was the problem responsible for most of the gynecologic consultations in the perimenopausal age group, thorough evaluation is a must especially in this age group to rule out endometrial cancer or its precursor lesion endometrial hyperplasia. Sonographic and histological assessment of the endometrium is the corner stone of diagnosis in the current practice $^{(5)}$.

Dysfunctional uterine bleeding (DUB) defined as abnormal uterine bleeding in the absence of underlying structural abnormalities. Obesity is the most prevalent nutritional disorder in which there is excessive storage of energy in the form of fat as per height, weight, race and gender. Obesity is prevalent among all age groups and is on the rise among adults especially the women worldwide in both developed and developing countries ${ }^{(6)}$.

Obesity has been suggested as a main underling risk factor for DUB. There was a strong association between obesity and DUB and therefore weight reduction should be considered as a conservative treatment besides of other medical or surgical treatment approaches ${ }^{(7)}$.

The prevalence of overweight and obesity among postmenopausal women of United Kingdom has been reported as $41 \%$ $(24 / 59)$ and $36 \%(21 / 59)$ respectively where as less than half of the premenopausal women $(48.27 \%, 14 / 29)$ of UK were observed as overweight/ obese by Begum et al. ${ }^{(8)}$. This figure was higher than statistics released by WHO in 2006 (WHO gobal infobase) which stated that the prevalence of overweight and obesity among postmenopausal women of U.K. was $30-40 \%$ and $25-31 \%$, respectively.

Rappelli (9) also reported that the prevalence of being overweight and obesity was higher in postmenopausal women. There was persistent debate regarding the most appropriate methods for investigating and managing PMB. Transvaginal sonography 
currently was considered to be the first-step technique to be performed in women with postmenopausal bleeding because it may reliably rule out endometrial cancer when endometrial thickness was less than $5 \mathrm{~mm}$ and because it was cost-effective. However, a thickened endometrium was a non-specific finding and additional tests are warranted. Most current protocols included the use of endometrial office biopsy. Histopathological diagnosis which was the gold standard for diagnosis, but it was expensive and need more preparation and may be done outpatient or inpatient like $\mathrm{D} \& \mathrm{C}$ procedure itself, although highly diagnostic, yet it had many limitations, including the need for general anesthesia, being a blind procedure with possible complications (10).

Endometrial biopsy is a blind procedure and should be considered part of the evaluation that could include imaging studies, such as hysteroscopy or transvaginal ultrasonography ${ }^{(11)}$.

In our study, we explored the relationship between body mass index and ultrasonographic endometrial thickness measurement and their correlation to theendometrial pathology in women of post menopausal bleeding. In the present study, 80 patients with postmenopausal bleeding were examined regarding BMI, ultrasonographic assessment of endometrial thickness and pathological findings of the endometrium. D\&C was performed for all patients under general anesthesia using a sharp ended curette starting first with the fundus, posterior wall, anterior wall, right and left lateral wall. The patient's age was ranged from 46-69 years, with a mean of $53.53 \pm 4.1$ years.

According to the pathology, the patients were categorized into 2 groups: the benign group: hyperplasia without atypia, hyperplastic endometrial polyp, disordered proliferative endometrium and atrophic endometrium). The malignant group: hyperplasia with atypia and endometrial carcinoma. According to the age, the malignant group had higher age compared to the benign group with a statistically highly significant difference in between both groups. Gravidity ranged from 2 to 7 , with a mean of $4.65 \pm 1.34$. Parity ranged from para 2 to para 7 , with a mean of $4.29 \pm 1.22$. There was a statistically significant difference between benign and malignant groups regarding gravidity and a statistically high significant difference between the two studied groups regarding parity, as gravidity and parity were higher in the benign group. Our study showed that there was a statistically significant difference between benign and malignant groups as regard age at menopause and a statistically high significant difference as regard duration of PMB; as the age at menopause was higher in malignant group and the duration of PMB was also longer in the malignant group.

In the present study, it was found that the range of BMI was from 28-44.9 with a mean of $32.99 \pm 3.3$. Malignant group had higher BMI compared to the benign group with a statistically highly significant difference between both groups.

Opolskiene et al. (12) determined whether endometrial volume or power Doppler indices as measured by three-dimensional (3D) ultrasound imaging can discriminate between benign and malignant endometrium, compared their diagnostic performance with that of endometrial thickness measurement using twodimensional (2D) ultrasound examination and determined whether power Doppler indices add any diagnostic information to endometrial thickness or volume. Median age was 65 years, and $24(39 \%)$ patients were on hormone replacement therapy. Patients with malignant endometrium tended to be older (median age, 73 years vs. 63 years), had a higher body mass index (median, $31 \mathrm{~kg} / \mathrm{m} 2$ vs. $27 \mathrm{~kg} / \mathrm{m} 2$ ) and thicker endometrium (median, $16.2 \mathrm{~mm}$ vs. 9.6 $\mathrm{mm}$ ) than those with benign endometrium, and fewer used hormone replacement therapy (23\% vs. $43 \%$ ).

Zhang et al. (13) showed that endometrial cancer risk was $32 \%$ higher in overweight (body mass index [BMI] 25-30) women and 2.5 times higher in obese (BMI 30+ women), both compared with healthyweight (BMI less than 25) women. But, Folsom et al. (14) found no association between abdominal body fat and the risk of endometrial carcinoma when adjusted for body mass index (BMI). On the other hand, Zaki et al. ${ }^{(15)}$ had shown in their study that abdominal obesity (waist circumference $>88 \mathrm{~cm}$ ), compared to general obesity (high BMI), was the best measure of obesity to be used in predicting the risk of endometrial cancer in Egyptian females with PMB.

Our study showed that the range of estradiol was from 3-35 with a mean of 20.43 \pm 
9.8. Benign group had higher estradiol level compared to benign group with a statistically highly significant difference between both groups. With regard to the ultrasonographic results in the present study, the inclusion criteria of the examined women included that endometrial thickness $(\mathrm{ET}) \geq 5 \mathrm{~mm}$; as most studies agree with that value to exclude endometrial pathology below it.

In the present study, there was a highly statistically significant difference between bengin and malignant groups as the endometrium was markedly thicker in the malignant group. Bruchim et al. (16) showed that the mean endometrial thickness for all patients without cancer was $(6.3 \pm 4.3)$ and it was significantly lower than that of patients with endometrial carcinoma ( $p<0.01$ ), whose endometrial thickness was more than $10 \mathrm{~mm}$, and no cases of endometrial carcinoma was found in any patients with endometrial thickness of less than $5 \mathrm{~mm}$. on the contrary, Phillip et al. ${ }^{(17)}$ suggested that the correlation between imaging and pathology was not reliable. Half of their cases with endometrial cancer had an endometrial thickness between $3 \mathrm{~mm}$ and $4 \mathrm{~mm}$. Seventy percent of the women with endometrial thickness greater than $5 \mathrm{~mm}$ had a benign pathology. Moodley and Roberts (18) chose endometrial thickness to be at least 4 $\mathrm{mm}$ in postmenopausal women as a limit value to exclude malignancy unless there are another risk factors. Also, in a study performed by Chandavarkar et al. ${ }^{(19)}$, no further diagnostic procedure should be done in a woman with postmenopausal bleeding and an endometrial echo complex $($ EEC) $\leq 4 \mathrm{~mm}$. However, an EEC $\leq 4 \mathrm{~mm}$ does not completely rule out endometrial cancer and cannot prevent histological evaluation.

Eitan et al. ${ }^{(20)}$ studied 29 patients with endometrial cancer to assess endometrial thickness as measured by ultrasound, which ranged from 5 to $32 \mathrm{~mm}$ (mean of $12 \mathrm{~mm}$ ); the mean in initial stages was $10 \mathrm{~mm}$ and the mean in advanced stages was $12 \mathrm{~mm}$. In this study, all patients diagnosed with cancer, and most patients diagnosed with endometrial hyperplasia, were at a cutoff point $\geq 10 \mathrm{~mm}$. our study displayed that there was a statistically high significant difference between bengin and malignant groups as regard the presence of fibroid uterus (due to presence of excess unopposed estrogen in those obese women) and the presence of adenomyotic or normal uterus in between both groups. Also, there was a highly statistically significant difference between bengin and malignant groups as the endometrial margin was more regular in the benign group, while it was more irregular in the malignant group and endometrium was heterogenous in only $49.3 \%$ in the benign group, but all the malignant group had heterogenous endometrium.

This study agrees with those of Opolskiene et al. ${ }^{(12)}$, as irregular surface of the endometrium has found to be a sign of endometrial malignancy and heterogeneous endometrial structure was a sign of endometrial cancer. With regard to the endometrial pathology in the present study, the most common endometrial pathology was endometrial hyperplasia followed by hyperplastic endometrial polyp, then disordered proliferative endometrium then hyperplasia with atypia and endometrial carcinoma. The presence of endometrial hyperplasia as the most common pathology was explained as most of the studied patients were obese with excess estrone derived from adipose tissue.

These findings are different in comparison to the following studies mostly due to different sample size and inclusion criteria. Yaman et al. (21) studied 213 cases of postmenopausal bleeding and found that 42 cases (19.7\%) were malignant, 109 cases $(51.17 \%)$ were benign lesions (polyphyperplasia), 62 cases $(29.1 \%)$ were atrophic. Opolskiene et al. ${ }^{(12)}$ studied 261 cases of postmenopausal bleeding and found that 63 cases $(24 \%)$ were malignant, 198 cases $(76 \%)$ were benign lesions. With regard the presence of endometrial polyp in the endometrial pathology; this study found that there was no statistically significant difference between benign and malignant groups as regard presence of endometrial polyp in endometrial pathology as it was present in only $21.7 \%$ of the benign group while $78.3 \%$ was absent, and it was absent in all the malignant group. This disagrees with a systematic review and meta-analysis done by Lee $\boldsymbol{e t}$ al. ${ }^{\left({ }^{22}\right)}$ that revealed that symptomatic vaginal bleeding and postmenopausal status in women with endometrial polyps are associated with an increased risk of endometrial malignancy. Costa-Paiva et al. ${ }^{(23)}$ also concluded in their study that women with endometrial polyps and postmenopausal bleeding had a higher prevalence of endometrial malignancy. In our study, by using ROC curve for estradiol in the prediction of pathological findings, the area under the curve is 
0.884 , sensitivity was $92 \%$ and specifcity was $88 \%$. Regarding body mass index in the prediction of pathological findings, the area under the curve is 0.796 , sensitivity was $80 \%$ and specificity was $77 \%$. Gupta et al. ${ }^{(24)}$ concluded that an endometrial thickness of $\geq 5 \mathrm{~mm}$ used as an indicator of endometrial pathology compared to uterine curettage has a sensitivity of $83 \%$, a specificity of $77 \%$, and a positive predictive value of $54 \%$. In a study used TVUS to correlate ET with histopathology, Buyuk $\boldsymbol{e t}$ al. (25) showed a sensitivity of $100 \%$ and specificity of $64 \%$ when a $6 \mathrm{~mm}$ cut off was used. All endometrial carcinomas in this group were found in patients with ET $\geq 7 \mathrm{~mm}$, which led the authors to suggest that it was reasonable not to perform dilatation and curettage if the ET $\leq 6 \mathrm{~mm}$. However, advanced endometrial carcinoma has been known to occur in cases without appreciable endometrial thickening on ultrasound. Karampl et al. (26) reported sensitivity, specificity, positive predictive value, negative predictive value of $33.3 \%$, $88.6 \%, 25 \%$ and $92.1 \%$ respectively, which shows less accuracy of TVS in diagnosis of uterine causes of perimenopausal bleeding, which may be related to machine used.

Moodley and Roberts ${ }^{(18)}$ were chosen endometrial thickness cut off value $4 \mathrm{~mm}$ in postmenopausal women as a limit value to exclude malignancy unless there were another risk factors. Results were found with sensitivity $100 \%$ and specificity $61 \%$. The positive and negative predictor values were $39 \%, 100 \%$ respectively. Timmermans et al. ${ }^{(27)}$ found that a threshold of $5 \mathrm{~mm}$ for the EEC had a sensitivity for endometrial cancer of only $90 \%$; $4 \mathrm{~mm}$, a sensitivity of $95 \%$ and $3 \mathrm{~mm}$, a sensitivity of $98 \%$. Correlating between different studied parameters revealed high significant correlations between estradiol and endometrial thickness, between age and parity and endometrial thickness, between gravidity and parity, between parity and endometrial thickness and between body mass index and endometrial thickness $(\mathrm{p}<0.001)$ and significant correlations between estradiol and age, parity and body mass index and between age and gravidity and body mass index ( $\mathrm{p}<$ 0.05). Phillip et al. (17) suggested that the correlation between imaging and pathology was not reliable. Half of their cases with endometrial cancer had an endometrial thickness between $3 \mathrm{~mm}$ and $4 \mathrm{~mm} .70 \%$ of the women with endometrial thickness greater than
$5 \mathrm{~mm}$ had a benign pathology. All patients with endometrial hyperplasia diagnosed on TVUS must have a thorough endometrial evaluation by $\mathrm{D} \& \mathrm{C}{ }^{(28)}$. In a small observational study, consisting of 20 women with DUB, assessed for obesity, Nouri et al. ${ }^{(7)}$ declared that two-third of studied women overweight status and other one-third cases were obese.

Viola et al. (29) determined the prevalence of endometrial hyperplasia and cancer in nonsymptomatic, overweight or obese premenopausal and postmenopausal women. They found serious endometrial pathology in overweight and obese asymptomatic women at reproductive age and at the postmenopause, which so far had not been described extensively. Obesity bears a high social price and represents elevated costs for the health-care system. The fact that the prevalence of obesity is increasing in most parts of the world and in view of the associated health risks, all possible interventions to reduce the weight of overweight and obese women through exercise and changes in dietary habits must be encouraged. Zaki $\boldsymbol{e t} \boldsymbol{a l}$. (15) assessed the independent effect of obesity on the probability to develop endometrial cancer in Egyptian females presenting with PMB. The study was conducted in Alexandria, Egypt and included all postmenopausal females presenting to the University Hospital of Gynecology and obstetrics with PMB. A questionnaire was completed, and data about anthropometric measurements including weight, height, and waist circumference were collected. Vaginal sonography, dilatation and curettage, and pathological examination were done by experts for all participants. Endometrial cancer was diagnosed in $38 \%$ of females presenting with PMB. Using ROC curve analysis, only the measure of abdominal obesity (waist circumference) showed significant accuracy in predicting endometrial cancer. The best cutoff point that maximizes accuracy was $88 \mathrm{~cm}$. Body mass index $(\geq 30$ vs. $\leq 30)$ showed no significant relation, and the ratio between upper and lower body obesity (W/H ratio) showed border line significant relation, whereas waist circumference ( $\geq 88$ vs. $\leq 88 \mathrm{~cm}$ ) showed strikingly high OR. The risk of abdominal obesity on endometrial cancer remains very high even after adjustment, in a logistic model, for other risk factors such as age at presentation, age at menarche, age at menopause, family history of malignancy, and gravidity. They 
concluded that obesity is a significant risk factor for the development of endometrial cancer. Abdominal obesity is the measure that best reflects this risk. Based on ROC analysis, they suggested a $\mathrm{WC} \geq 88 \mathrm{~cm}$ as the appropriate cutoff point to identify the risk category for the development of endometrial cancer in Egyptian females with PMB. Abd Ali and Al-Zaidi (30) examined the correlation between BMI, lipid profile and estradiol in diabetic pre-ovulatory women as a sample of Iraqi Asian women. They concluded that Iraqi diabetic pre menopausal women at their pre ovulatory period exhibit a proatherogenic risk profile because of their abnormal BMI, higher LDL-C, lower HDL-C and estradiol levels.

\section{CONCLUSION}

All patients with postmenopausal bleeding must be properly assessed to rule out the possibility of malignancy. Obese postmenopausal women had increased risk for endometrial cancer. The most common pathology found in patients with postmenopausal bleeding in this study was endometrial hyperplasia and this was explained by excess estrone derived from excess adipose tissue as most patients were obese.

\section{REFERENCES}

1. Goodman A (2014): Postmenopausal uterine bleeding. UpToDate. available at: https://www.uptodate.com/contents/postm enopausal-uterine-bleeding

2. Friedenreich C, Cust A, Lahmann PH et al. (2007): Anthropometric factors and risk of endometrial cancer. Cancer Causes Control, 18: 399-413.

3. Feldman S (2014): Evaluation of the endometrium for malignant and premalignant disease. UpToDate. Available at: https:// www. uptodate. com/ contents/ evaluation-of-the-endometriumfor-malignant-or-premalignant-disease

4. Sharma S, Bakshi R, Tandon VR et al. (2008): Postmenopausal obesity. J.K. Science, 10(3): 105-106.

5. Pillai SS (2016): Sonographic and histopathological correlation and evaluation of endometrium in perimenopausal women with abnormal uterine bleeding. International Journal of Reproduction, Contraception, Obstetrics and Gynecology, 3(1):113-7.

6. Flegal KM (2005): Epidemiologic aspects of overweight and obesity in the United
States. Physiology \& behavior, 86(5):599602.

7. Nouri M, Tavakkolian A and Mousavi SR (2014): Association of dysfunctional uterine bleeding with high body mass index and obesity as a main predisposing factor. Diabetes Metab. Syndr., 8(1): 1-2.

8. Begum $P$, Richardson $\mathrm{CE}$ and Carmichael AR (2009): Obesity in post menopausal women with a family history of breast cancer: prevalence and risk awareness. Int .Semin. Surg. Oncol., 6: 1-9.

9. Rappelli A (2002): Hypertension and obesity after the menopause. J. Hypertens., 20(2): 26-28.

10. Trimble $\mathbf{C L}$, Kauderer $\mathbf{J}$ and Zaino $R$ (2006): Concurrent endometrial carcinoma women with a biopsy diagnosis of atypical endometrial hyperplasia. Cancer, 106: 812819.

11. Dimitraki M, Tsikouras P, Bouchlariotou S et al. (2010): Clinical evaluation of women with PMB. Is it always necessary an endometrial biopsy to be performed? A review of the literature. Archives of Gynecology and Obstetrics, 283(2): 261-266.

12. Opolskiene G, Sladkevicius P, Jokubkiene L et al. (2010): Threedimensional ultrasound imaging for discrimination between benign and malignant endometrium in women with postmenopausal bleeding and sonographic endometrial thickness of at least $4.5 \mathrm{~mm}$. Ultrasound Obstet. Gynecol., 35: 94-102.

13. Zhang $Y$, Liu $H$ and Yang $S$ (2013): Overweight, obesity and endometrial cancer risk: results from a systematic review and meta-analysis. Int. J. Biol. Markers, 29(1): 21-9.

14. Folsom AR, Kaye SA, Potter JD et al. (1999): Association of incident carcinoma of the endometrium with body weight and fat distribution in older women. Cancer Gynecologic Oncology, 72: 138-142.

15. Zaki A, Gaber A, Ghanem E et al. (2011): Abdominal obesity and endometrial cancer in Egyptian females with postmenopausal bleeding. Nutrition and Cancer, 63(8): 1272-1278.

16. Bruchim I, Biron-Shental T, Altaras MM et al. (2004): Combination of endometrial thickness and time since menopause in predicting endometrial cancer in women with 
postmenopausal bleeding. Journal of Clinical Ultrasound, 32(5):219-24.

17. Phillip H, Dacosta $V$ and Fletecher $H$ (2004): Correlation between transvaginal ultrasound measured endometrial thickness and histopathological finding in AfroCaribbean Jamican women with postmenopasual bleeding. J. Obstet. Gynecol.,24: 268-272.

18. Moodley $M$ and Roberts $C$ (2004): Clinical pathway for the evaluation of postmenopausal bleeding with an emphasis on endometrial cancer detection. J. Obstet. Gynaecol., 24(7): 736-741.

19. Chandavarkar U, Kuperman JM, Muderspach LI et al. (2013): Endometrial echo complex thickness in postmenopausal endometrial cancer. Gynecol. Oncol., 131(1): 109-112.

20. Eitan R, Saenz CC, Venkatraman ES et al. (2005): Pilot study prospectively evaluating the use of the measurement of preoperative sonographic endometrial thickness in postmenopausal patients with endometrial cancer. Menopause, 12(1): 2730.

21. Yaman C, Habelsberger A, Tews G et al. (2008): The role of three-dimensional volume measurement in diagnosing endometrial cancer in patients with postmenopausal bleeding. Gynecologic Oncology, 110: 390-395.

22. Lee SC, Kaunitz AM, Sanchez-Ramos L et al. (2010): The oncogenic potential of endometrial polyps: a systematic review and metaanalysis. Obstet Gynecol., 116: 1197-1205.

23. Costa-Paiva L, Godoy CE, Antunes A et al. (2011): Risk of malignancy in endometrial polyps in premenopausal and postmenopausal women according to clinicopathologic characteristics. The Journal of The North American Menopause Society, 18(12): 1278-1282.

24. Gupta JK, Wilson S, Desai P et al. (1996): How should we investigate women with postmenopausal bleeding? Acta Obstet Gynecol Scand., 75:475-479.

25. Buyuk E, Durumusoglu F, Erenus $M$ (1999): Endometrial disease diagnosed by transvaginal ultrasound and dilatation and curettage. Acta Obstet Gynecol Scand., 78 (5): 419-22.

26. Karampl EM, Bourne T, Hurler S et al. (2001): Transvaginal ultrasonography, sonohysterography and operative hysteroscopy for the evaluation of abnormal uterine bleeding.Acta Obstet Gynecol Scand., 80(7): 616-622.

27. Timmermans BCO, Khan KS, Bachmann LM et al. (2010): Endometrial thickness measurement for detecting endometrial cancer in women with postmenopausal bleeding. Am J Obstet Gynecol., 116: 160-7.

28. Doraiswami S, Johnson T, Rao $\mathrm{S}$ et al. (2011): Study of Endometrial Pathology in Abnormal Uterine Bleeding. J Obstet Gynaecol India., 61(4): 426-30.

29. Viola AS, Gouveia D, Andrade L et al. (2008): Prevalence of endometrial cancer and hyperplasia in non-symptomatic overweight and obesity women. Australian and New Zealand Journal of Obstetrics and Gynaecology, 48: 207-213.

30. Abd Ali ZA and Al-Zaidi MS (2011): The association between body mass index, lipid profile and serum estradiol levels in a sample of Iraqi diabetic premenopausal women. Oman Medical Journal, 26(4): 263-266. 